\title{
Correction to: "SPOCU": scaled polynomial constant unit activation function
}

\author{
Jozef Kisel'ák ${ }^{1,2} \cdot$ Ying Lu $^{3}$ · Ján Švihra ${ }^{4}$ Peter Szépe ${ }^{5}$ Milan Stehlík ${ }^{2,6,7}$ (1)
}

Published online: 17 November 2020

(c) Springer-Verlag London Ltd., part of Springer Nature 2020

\section{Correction to: Neural Computing and Applications https://doi.org/10.1007/ s00521-020-05182-1}

\section{Introduction}

Here we provide Addendum on SPOCU fitting and Erratum to Article Title:"SPOCU": scaled polynomial constant unit

The original article can be found online at https:// doi.org/10.1007/s00521-020-05182-1.

\section{Milan Stehlík}

Milan.Stehlik@jku.at

Jozef Kisel'ák

jozef.kiselak@upjs.sk

Ying Lu

ylu1@stanford.edu

Ján Švihra

svihra@jfmed.uniba.sk

Peter Szépe

peter.szepe@atlas.sk

1 Institute of Mathematics, Faculty of Science, P.J.Šafárik University in Košice, Kosice, Slovak Republic

2 Linz Institute of Technology (LIT) and Department of Applied Statistics, Johannes Kepler University in Linz, Linz, Austria

3 School of Medicine, Stanford University, Stanford, USA

4 Department of Urology, Jessenius Faculty of Medicine, Comenius University Bratislava, 03659 Martin, Slovak Republic

5 Department of Pathological Anatomy, University Hospital Martin, Kollárova 2, 03659 Martin, Slovak Republic

6 Department of Statistics, University of Valparaíso, Valparaíso, Chile

7 Department of Statistics and Actuarial Science, The University of Iowa, Iowa City, USA activation function. https://doi.org/10.1007/s00521-02005182-1.

Following the publication of our article [1], we have become aware that the legends of Fig. $8 b$ should read as follows: generator $h(\cdot)$ of the activation function $S$. Moreover, the following Fig. 1 provides the graph of activation function $S(c=\propto)$.

The SPOCU activation function is given by

$s(x)=\alpha h\left(\frac{x}{\gamma}+\beta\right)-\alpha h(\beta)$

where $\beta \in(0,1), \alpha, \gamma>0$ and

$h(x)=\left\{\begin{array}{cc}r(c), & x \geq c, \\ r(x), & x \in[0, c), \\ 0, & x<0,\end{array}\right.$

with $r(x)=x^{3}\left(x^{5}-2 x^{4}+2\right)$ and $1 \leq c<\propto$ (we admit $c$ goes to infinity with $r(c) \rightarrow \propto$ ).

Shortly after publication of our article [1], we have received questions from the community how to implement SPOCU, since it is not involved in current software packages, e.g. in Matlab or Python (Keras). Here we provide a short idea how to select parameters $\alpha, \beta, \gamma$ or $c$.

Parametric space for parameters of SPOCU is $(\alpha, \beta, \gamma$, $c) \in(0, \propto) \times(0,1) \times(0, \propto) \times(1, \propto]:=\mathrm{P}$. For proper choice of the parameters, it is sufficient they belong to $\mathrm{P}$ and they can satisfy several conditions. One set of conditions has been outlined in [1], which gives ${ }^{1}$

$\mathrm{E} f[s(z)]=: \operatorname{Mean}(\alpha, \beta, c, \gamma)=0$

$\mathrm{E} f\left[s^{2}(z)\right]=: \operatorname{Var}(\alpha, \beta, c, \gamma)=1$

$\|J(\alpha, \beta, c, \gamma)\|<1$

hold. Here $f(z)$ is the pdf of underlying distribution, $J$ is the Jacobi matrix w.r.t to mean and variance parameters and I. $\|$ is the appropriate matrix norm, e.g. $L_{1}$ or $L_{2}$, as considered in the application.

\footnotetext{
${ }^{1}$ Standard normality implies pdf of the form $f(z)=\frac{1}{\sqrt{2 \pi}} \mathrm{e}^{-\frac{z^{2}}{2}}$.
} 


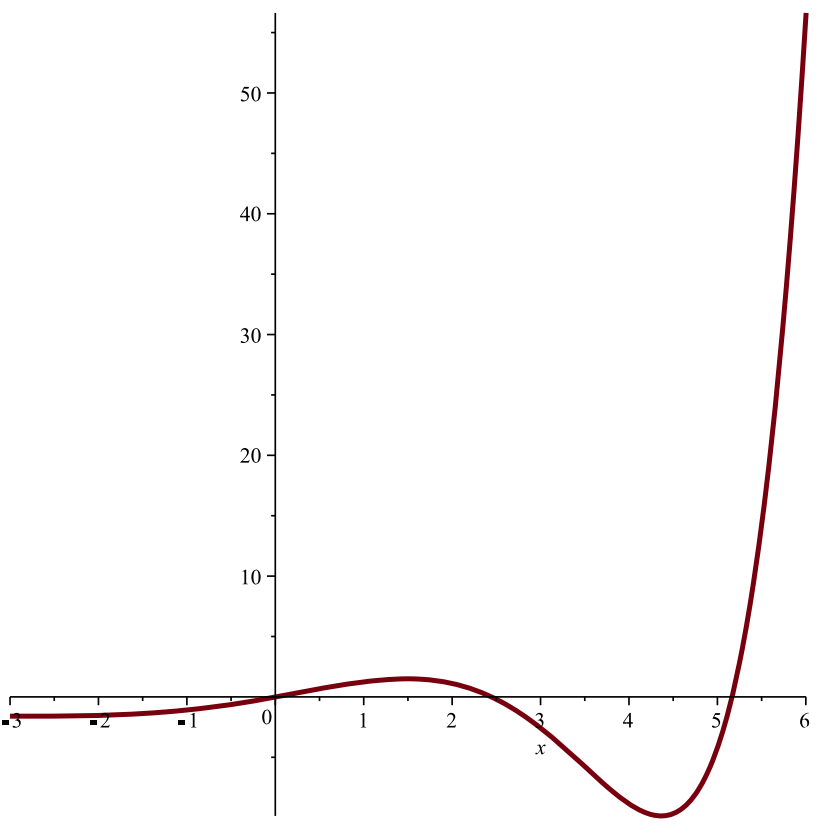

Fig. 1 Activation function $S$

However, other sets of conditions can be more attractive for the reader, all depends on the aims and type of network.
E.g. one can, similarly as in 5.1. of [1] fix $c=\gamma=1$. And, to compute $\alpha, \beta$ one can consider Pareto density $g(x)=a(x+1)^{-a-1}, x>0, a>1$ to satisfy for some $a>1$

$\mathbb{E}_{g}[s(x)]=\frac{1}{a-1}$

with additional condition $s^{\prime}(0)=1$, which is $2\left(\beta^{2} \beta^{5}-\beta^{4}+3\right)=\gamma$. Thus, for Pareto tail parameter $a=4$ we received in Maple software $\alpha=0.8874425243$ and $\beta=0.4495811364$.

Acknowledgements Authors acknowledge professional support of Editor in Chief, Professor John Macintyre.

\section{Reference}

1. Kisel'ák J, Lu Y, Švihra J, Szépe P, Stehlík M (2020) "SPOCU”: scaled polynomial constant unit activation function. Neural Comput Appl. https://doi.org/10.1007/s00521-020-05182-1

Publisher's Note Springer Nature remains neutral with regard to jurisdictional claims in published maps and institutional affiliations. 